This item was submitted to Loughborough's Research Repository by the author.

Items in Figshare are protected by copyright, with all rights reserved, unless otherwise indicated.

\title{
Electrospinning of polylactic acid fibres containing tea tree and manuka oil
}

PLEASE CITE THE PUBLISHED VERSION

http://dx.doi.org/10.1016/j.reactfunctpolym.2017.06.013

\section{PUBLISHER}

(c) Elsevier

VERSION

AM (Accepted Manuscript)

\section{PUBLISHER STATEMENT}

This work is made available according to the conditions of the Creative Commons Attribution-NonCommercialNoDerivatives 4.0 International (CC BY-NC-ND 4.0) licence. Full details of this licence are available at: https://creativecommons.org/licenses/by-nc-nd/4.0/

\section{LICENCE}

CC BY-NC-ND 4.0

\section{REPOSITORY RECORD}

Zhang, Wanwei, Chao Huang, Olga E. Kusmartseva, Noreen L. Thomas, and Elisa Mele. 2017. "Electrospinning of Polylactic Acid Fibres Containing Tea Tree and Manuka Oil". figshare. https://hdl.handle.net/2134/26259. 


\title{
Electrospinning of polylactic acid fibres containing tea tree and manuka oil
}

\author{
Wanwei Zhang ${ }^{\mathrm{a}}$, Chao Huang ${ }^{\mathrm{a}}$, Olga Kusmartseva ${ }^{\mathrm{b}}$, Noreen L. Thomas ${ }^{\mathrm{a}}$, Elisa Mele ${ }^{\mathrm{a}, *}$ \\ a Department of Materials, Loughborough University, LE11 3TU Loughborough, United Kingdom \\ ${ }^{\mathrm{b}}$ Department of Physics, Loughborough University, LE11 3TU Loughborough, United Kingdom
}

\section{A R T I C L E IN F O}

Keywords:

Electrospinning

Essential oils

Polylactic acid

\begin{abstract}
A B S T R A C T
Here the effect of tea tree and manuka essential oils (EOs) on the mechanical properties and antibacterial activity of electrospun polylactic acid (PLA) fibres is investigated. It is found that the essential oils work as plasticisers for PLA, lowering the glass transition temperature of the resulting composite fibres up to $60 \%$ and increasing elongation-at-break and tensile strength up to 12 times. Manuka EO is particularly successful in blocking the formation of biofilms of Staphylococcus epidermidis that is typically involved in nosocomial infections associated with implanted devices. The results demonstrate that natural extracts can be used to control the mechanical behaviour of PLA fibres and to confer antibacterial activity.
\end{abstract}

\section{Introduction}

The recent health concerns associated with the side effects of synthetic additives used in pharmacy, cosmetics, agriculture and the food industry have stimulated a consumer demand for natural alternatives [1]. There is therefore a growing interest in plant extracts with biological activity, such as essential oils (EOs). They are complex mixtures of volatile compounds that possess, among other characteristics, antimicrobial, antioxidant, anti-inflammatory, antifungal and anticancer properties [2].

Nanostructured systems containing EOs, mainly produced by electrospinning, have so far been proposed for wound management and food packaging applications [3-16]. For instance, electrospun scaffolds made of a blend of chitosan/poly(ethylene oxide) (PEO) and cinnamon essential oil have been developed for the treatment of infections caused by Escherichia coli and Pseudomonas aeruginosa [3]. The efficacy against E. coli of cinnamon oil has also been demonstrated when cellulose acetate was used as the polymer matrix for nanofibrous dressings [4]. Moreover, in the food sector the antibacterial activity of cinnamon EO has been exploited to fabricate packaging materials that avoid microbial contamination of food and consequently food spoilage [5]. Cinnamon $\mathrm{EO}$ and $\beta$-cyclodextrin inclusion complex were incorporated into polylactic acid (PLA) fibres, and the inhibition of the growth of E. coli and Staphylococcus aureus was analysed. Another EO that has been electrospun is lavender oil. Nanofibers of polyacrylonitrile (PAN) [6] and

\footnotetext{
* Corresponding author.

Email address: E.Mele2@lboro.ac.uk (E. Mele)
}

sodium alginate [7] combined with lavender EO were used for preventing bacterial colonisation of skin wounds ( $S$. aureus and Klebsiella pneumonia). Furthermore, it was demonstrated that dressings made of sodium alginate and lavender oil exhibit anti-inflammatory properties, reducing the production of pro-inflammatory cytokines both in vivo and in vitro [7]. Electrospun fibres containing thyme [8], peppermint and lemongrass have also been produced [4].

Recently, essential oils have been incorporated into electrospun fibres not only to achieve bioactivity but also to control the thermal properties of the resulting mats $[17,18]$. Souza et al. have reported $18 \%$ reduction of the glass transition temperature $\left(T_{g}\right)$ of PLA fibres when $20 \%$ of Linalool was used [17]. In another study, the incorporation of Candeia EO into PLA fibres induced a $T_{g}$ reduction of 30\% (15\% of EO) [18]. In both cases, the natural extracts act as plasticisers for PLA, affecting the mobility of the polymer chains and lowering chain-to-chain interactions, as also demonstrated for PLA-EOs films [19].

In this study, the electrospinning of PLA fibres containing essential oils derived from Melaleuca alternifolia (Australian Tea Tree oil) and Leptospermum scoparium (New Zealand manuka oil) is demonstrated. The EOs extracted from these two different species of Myrtaceae are typically used in aromatherapy and for the treatment of microbial infections. This study investigates the effect that diverse EO concentrations (from 2.5 to $15.0 \% \mathrm{v} / \mathrm{v}$ ) have on fibre morphology, thermal and mechanical properties. It is shown that both oils are strong plasticisers for PLA. The glass transition temperature $\left(T_{g}\right)$ of PLA fibres containing Tea Tree and Manuka EO was up to $40{ }^{\circ} \mathrm{C}$ lower than the $T_{g}$ of pristine PLA fibres. Differently from previous studies $[17,18]$, the mechanical 
properties of the composite fibres are also characterised, demonstrating that both elongation at break and tensile strength at break increase by increasing the amount of EO, with values that are up to 12 times higher than those of PLA fibres without EO. PLA/Manuka fibres also possess antibacterial activity, being able to inhibit the growth of Staphylococcus epidermidis, which is one of the main causes of biofilm formation on implanted medical devices [20]. The mechanical properties and the bioactivity of the composite PLA/EO mats make them promising as infection-resistant materials for biomedical applications.

\section{Experimental section}

\subsection{Electrospinning process}

Solutions for electrospinning were prepared by dissolving PLA (Ingeo $^{\text {TM }} 4060$ D supplied by Natureworks LLC) in acetone at $15 \% \mathrm{w} / \mathrm{v}$ concentration. PLA 4060D is an amorphous polymer with an L-lactide content of around $88 \mathrm{wt} \%$ and a weight average molecular weight $\left(\mathrm{M}_{\mathrm{w}}\right)$ as determined by gel permeation chromatography (GPC) of $1.15 \times 10^{5} \mathrm{~g}$ / mol. The optimum concentration for defect-free nanofibre production is between 2 and 2.5 times the critical chain entanglement concentration $\left(C_{e}\right)$ and the value of $C_{e}$ for PLA/acetone was found to be $6 \% \mathrm{w} / \mathrm{v}$ $[21,22]$. Hence the concentration of $15 \% \mathrm{w} / \mathrm{v}$ was chosen.

Tea Tree oil (Melaleuca alternifolia) or manuka oil (Leptospermum scoparium) were added to the PLA/acetone solution at different concentrations: $2.5,5.0,7.5,10.0$ and $15.0 \% \mathrm{v} / \mathrm{v}$. Tea Tree oil is mainly constituted by terpinen-4-ol, $\gamma$-terpinene, $\alpha$-terpinene and 1,8-cineole; Manuka oil contains triketones, sesquiterpenes and p-cymene [23-25]. PLA, PLA/Tea Tree EO (PLA/TT EO) and PLA/Manuka EO (PLA/M EO) solutions were loaded into a $3 \mathrm{~mL}$ plastic syringe capped with one 18-gauge needle. The solutions were injected at a constant flow rate of $2 \mathrm{ml} / \mathrm{h}$ that was generated by a pump system (PHD ULTRA, Harvard Apparatus). A voltage of $18.5 \mathrm{kV}$ was applied between the needle and an aluminium planar collector, placed at a relative distance of $15 \mathrm{~cm}$. The electrospinning process was conducted at $20^{\circ} \mathrm{C}$, using the Spraybase ${ }^{\circledR}$ Electrospinning equipment.

\subsection{Characterisation of the fibrous mats}

The morphology of the electrospun fibres was observed using a scanning electron microscope (SEM), with a field emission gun system LEO1530VP. A gold/palladium coating was deposited onto the fibrous mats before imaging using an Emitech SC7640 sputter coater (90 s sputter time).

The chemical analysis of the fibres with and without the essential oils, and of the pristine essential oils was carried using Raman spectroscopy. Raman measurements were performed in a confocal back-scattering configuration with a HORIBA LabRAM HR spectrometer equipped with an Olympus microscope, a liquid-nitrogen cooled Charge-Coupled Detector and a He-Ne laser (excitation wavelength of $633 \mathrm{~nm}$ ). The laser power on the sample surface was $3.75 \mathrm{~mW}$.

Thermal characterisation of the mats was conducted using differential scanning calorimetry (DSC Q200, TA Instruments Calorimetric Analyser, USA) in a nitrogen atmosphere with a flow rate of $50 \mathrm{ml} / \mathrm{min}$. Approximately $8 \mathrm{mg}$ of each type of mat was sealed in an aluminium pan and heated from -20 to $100{ }^{\circ} \mathrm{C}$ at $10^{\circ} \mathrm{C} / \mathrm{min}$. Empty aluminium pans were used as reference. Data were analysed using the TA universal analysis software. All the reported values are the average of 3 samples.

For analysis of mechanical properties, the fibrous mats were detached from the aluminium foil and cut using an ISO527-2/5A die. Tensile tests were carried out using a single column table top Instron system at room temperature. The rate of extension was set at $2 \mathrm{~mm} /$ min for the preload stage and at $3 \mathrm{~mm} / \mathrm{min}$ for the test. Side action grip clamps with flat jaw faces were used.

\subsection{Antibacterial tests}

The antibacterial activity of the nanofibrous mats containing Tea Tree oil or Manuka oil, was tested against Staphylococcus epidermidis. PLA nanofibers without essential oils were used as control. Bacterial cultures were prepared in $2.5 \mathrm{mg} / 100 \mathrm{ml}$ water $\mathrm{LB}$ broth, according to the manufacturer's instructions, and incubated at $37{ }^{\circ} \mathrm{C}$ overnight. $11.1 \mathrm{mg}$ LB Agar was dissolved in $300 \mathrm{ml}$ of water and then heated at $120{ }^{\circ} \mathrm{C}$ for $15 \mathrm{~min}$. Agar plates were then prepared and inoculated with $1 \mathrm{ml}$ of bacteria culture. The electrospun mats (weight $45 \mathrm{mg}$ ) were placed on top of the plates and incubated at $37{ }^{\circ} \mathrm{C}$ for two days. Photos of the plates were taken and the zone of inhibition was measured.

\section{Results and discussion}

The solubility of PLA, Tea Tree oil and Manuka oil in acetone allowed homogeneous solutions to be obtained, which gave good dispersion of the essential oils in the polymer matrix. This had a positive effect on the morphology of the electrospun fibres.

As shown in Fig. 1, SEM analysis of the fibrous mats revealed the absence of defects and beads. Fibres produced from PLA solution without EOs (Fig. 1a) showed a cylindrical and well-defined shape, with overlapping but not merged fibres. The addition of $5 \% \mathrm{v} / \mathrm{v}$ of Tea Tree EO to PLA/acetone solution gave rise to the formation of junctions where fibres merged together, as shown in Fig. 1b and highlighted by the blue circle in the inset of Fig. 1b. The extent of this inter-fibre bonding further increased with the increase of Tea Tree EO concentration, as seen in the inset of Fig. 1c for PLA fibres elecrospun from solutions containing $10 \% \mathrm{v} / \mathrm{v}$ of Tea Tree EO. Two or more fibres have fused together and formed bundles. When the concentration of EO reached 15\% v/v, mats with highly interconnected fibres were produced. The fibrous structure is still visible but fibres have merged together and lost their cylindrical shape (Fig. $1 \mathrm{~d}$ and inset).

The effect of oil concentration was less evident for fibres produced from PLA/Manuka EO solutions (Fig. 1e-g). Only at EO concentration of $15 \% \mathrm{v} / \mathrm{v}$, the morphology of the fibrous mat changed, with extended regions of contact between fibres but without fusion (Fig. $1 \mathrm{~g}$ and relative inset). The phenomenon of fibre coalescence has been already reported in the literature. For example, interconnected networks of Fe-N/ $\mathrm{C}$ Polyvinylpyrrolidone can be formed as a consequence of water absorption [26]; Electrospun fibres of nitrile butadiene rubber tend to fuse together if the crosslinking degree is insufficient [27]. In the case of PLA and EOs, the changes observed in fibre morphology can be attributed to the thermal properties of the composite fibres, as discussed below.

The chemical properties of the mats were characterised by Raman spectroscopy. The Raman spectra of pure Tea Tree (dashed green curve) and Manuka essential oil (dashed magenta curve), and the spectra of the pure PLA fibres (continuous grey curve), PLA/TT EO (continuous green curve) and PLA/M EO (continuous magenta curve) fibres are shown in Fig. 2. The spectrum of PLA exhibits the characteristic band of $\mathrm{C}=$ $\mathrm{O}$ with one peak at $1770 \mathrm{~cm}^{-1}$ and a shoulder at $1754 \mathrm{~cm}^{-1}$ [28]. The peaks at $1454 \mathrm{~cm}^{-1}, 1387 \mathrm{~cm}^{-1}$ and $1129 \mathrm{~cm}^{-1}$ are assigned to $\mathrm{CH}_{3}$ asymmetric and symmetric wagging, and bending modes respectively; whereas, the peaks at $1182 \mathrm{~cm}^{-1}$ and $1096 \mathrm{~cm}^{-1}$ correspond to the OCO stretching mode. The $\mathrm{CH}$ bending, $\mathrm{C}^{-} \mathrm{CH}_{3}$ stretching, C-COO stretching and $\mathrm{C}=\mathrm{O}$ deformation appear at $1297 \mathrm{~cm}^{-1}, 1045 \mathrm{~cm}^{-1}$, $875 \mathrm{~cm}^{-1}$, and $745 \mathrm{~cm}^{-1}$, respectively [29].

The Raman spectrum of Tea Tree oil (Melaleuca alternifolia) in Fig. $2 \mathrm{a}$ is characterised by bands assigned to terpinen-4-ol, which is one of the main components of this essential oil [23]. Particularly, the peaks 
(a)

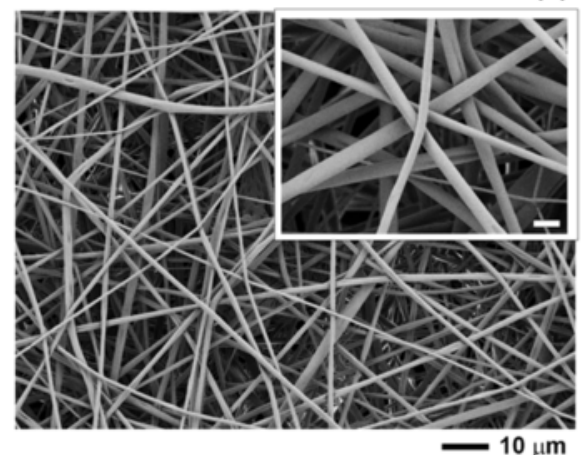

(b)

(e)
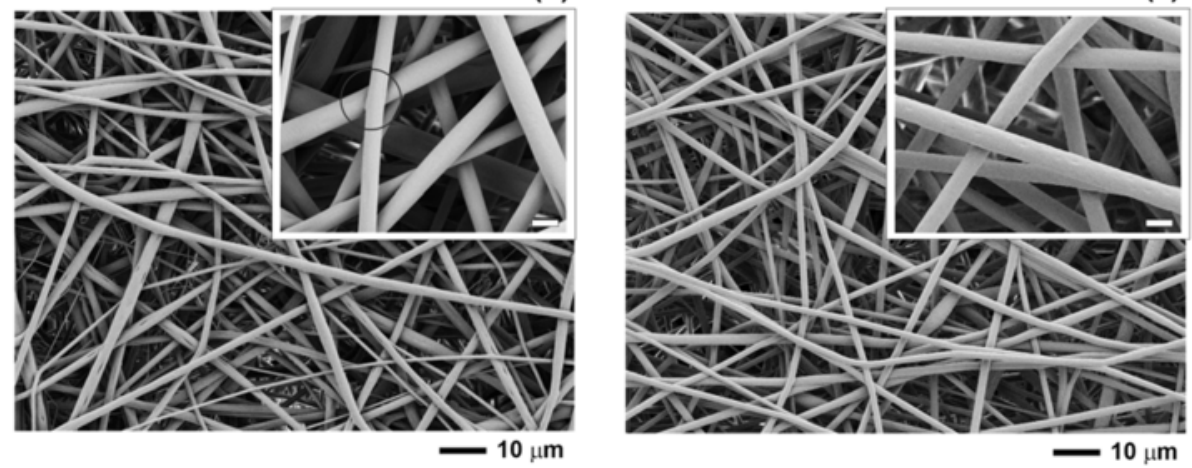

(c)
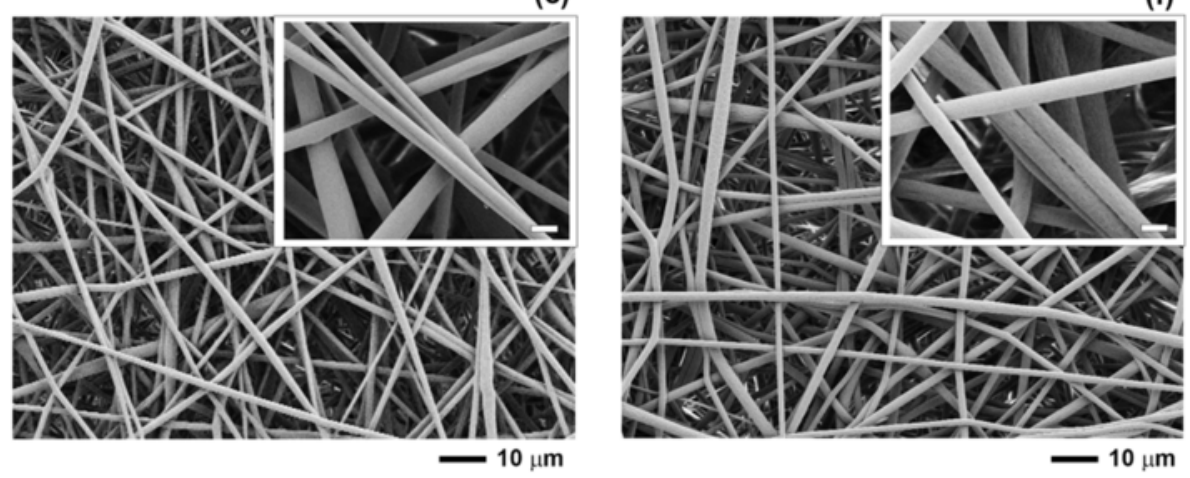

(d)
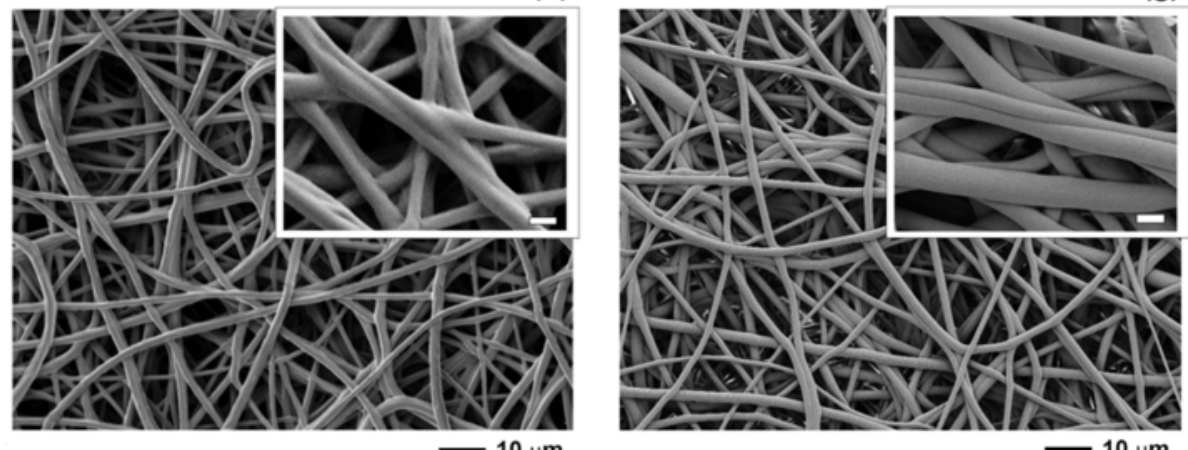

(g)

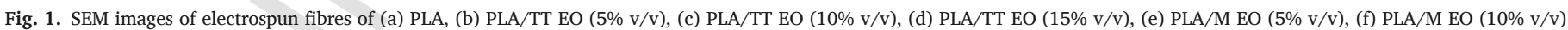
and $(\mathrm{g})$ PLA/TT EO $(15 \% \mathrm{v} / \mathrm{v})$. Insets: SEM images at higher magnification. Scale bar $=2 \mu \mathrm{m}$.

at $1678 \mathrm{~cm}^{-1}, 1376 \mathrm{~cm}^{-1}, 1307 \mathrm{~cm}^{-1}$ and $730 \mathrm{~cm}^{-1}$ correspond to $\mathrm{C}$ $=\mathrm{C}$ stretching modes of terpinen-4-ol. The presence of $\gamma$-terpinene and $\alpha$-terpinene, which are two other components of Tea Tree EO, is confirmed by the peaks at $1702 \mathrm{~cm}^{-1}(\mathrm{C}=\mathrm{C}$ stretching of $\gamma$-terpinene), $1612 \mathrm{~cm}^{-1}$ ( $\mathrm{C}=\mathrm{C}$ stretching of $\alpha$-terpinene), $1425 \mathrm{~cm}^{-1}$ (C
$-\mathrm{H}$ bending of $\gamma$-terpinene), $756 \mathrm{~cm}^{-1}$ (ring deformation of $\gamma$-terpinene). The bands at $1445 \mathrm{~cm}^{-1}$ and $652 \mathrm{~cm}^{-1}$ are assigned to $\mathrm{CH}_{3} / \mathrm{CH}_{2}$ bending mode and to 1,8-cineole, respectively. The analysis of the PLA/TT EO fibres reveals the presence of the characteristic peaks of PLA and a reduction of the intensity of some of the bands of Tea 
(a)

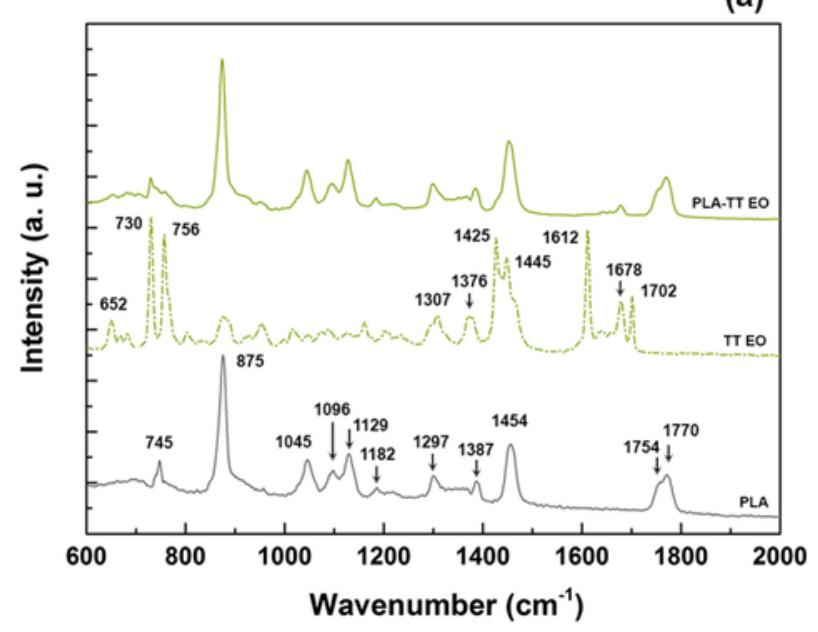

(b)

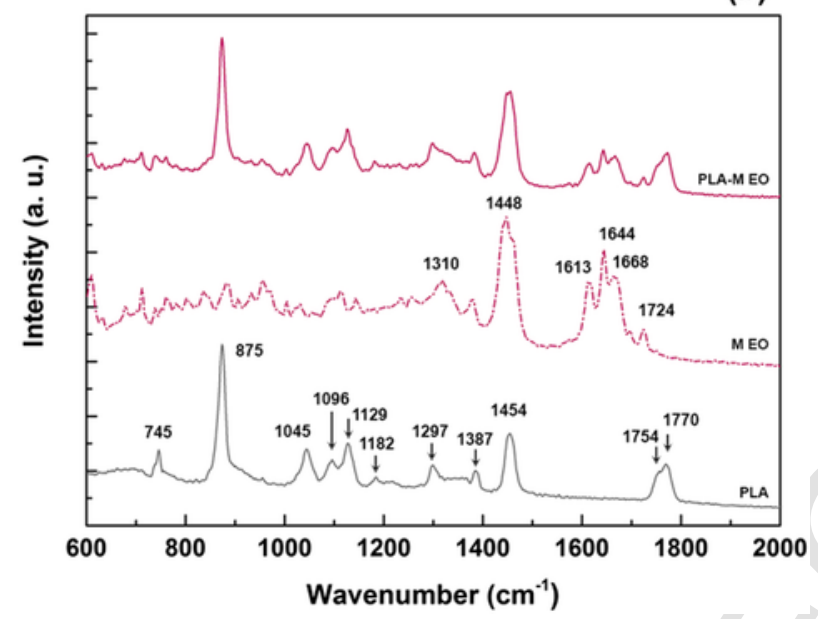

Fig. 2. Comparison of the Raman spectra of PLA electrospun fibres (continuous grey line), essential oils and composite PLA/EOs fibres: (a) pristine Tea Tree oil (dashed green line) and PLA/TT EO (10\% v/v) fibres (continuous green line); (b) pristine Manuka essential oil (dashed magenta line) and PLA/M EO (10\% v/v) fibres (continuous magenta line). (For interpretation of the references to color in this figure legend, the reader is referred to the web version of this article.)

Tree oil, particularly those associated with $\gamma$-terpinene and $\alpha$-terpinene. This can be due to the evaporation of the oil during the electrospinning procedure. The bands of terpinen-4-ol are visible.

The main constituents of $M$. alternifolia oil are not present in Manuka essential oil (Leptospermum scoparium from New Zealand), for which the relevant compounds are flavonoids, triketones (such as leptospermone and flavesone), sesquiterpenes ( $\alpha$-copaene and calamenene) and monoterpenes ( $\alpha$-pinene, myrcene, and $p$-cymene) [24,25]. In Fig. 2b, the Raman spectrum of the Manuka EO exhibits bands of triketones at $1724 \mathrm{~cm}^{-1}, 1668 \mathrm{~cm}^{-1}, 1448 \mathrm{~cm}^{-1}$, and $1310 \mathrm{~cm}^{-1}[30,31]$. The peaks at $1644 \mathrm{~cm}^{-1}$ and $1613 \mathrm{~cm}^{-1}$ can be ascribed to $\mathrm{C}=\mathrm{C}$ stretching mode and ring stretching mode of $p$-cymene, respectively [32]. Those bands are still visible in the spectrum of PLA/M EO electrospun mats together with the peaks of PLA. Therefore, Manuka oil and particularly triketones, which are responsible for the antibacterial activity of this EO [31], are contained inside the fibres.

DSC analysis was performed in order to evaluate the glass transition temperature $\left(T_{g}\right)$ of PLA, PLA/TT EO and PLA/M EO fibres. As shown in Fig. 3, the $T_{g}$ of PLA fibres (grey bar) was $(53 \pm 1)^{\circ} \mathrm{C}$, in agreement with previous reports [33]. However, PLA/TT EO (light green bars)

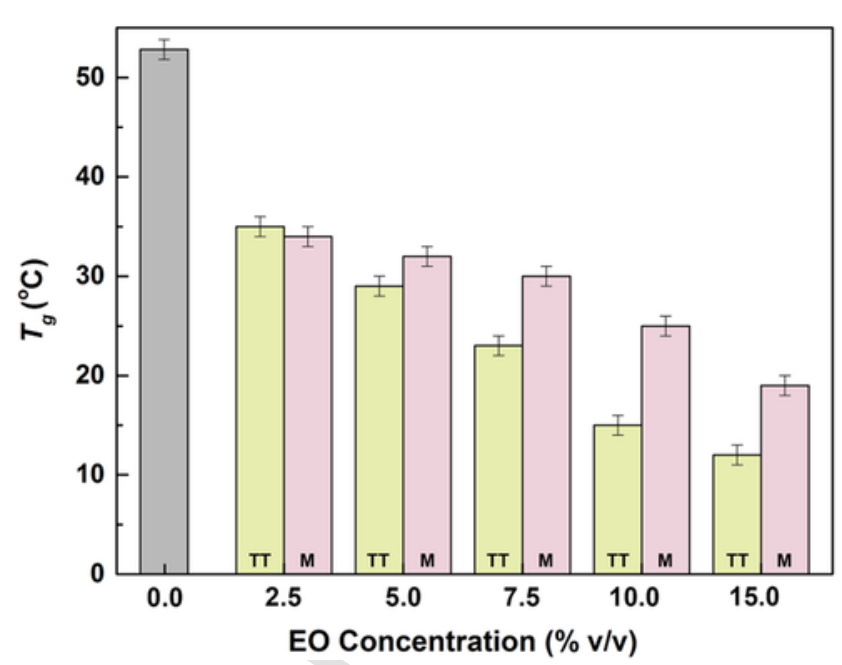

Fig. 3. Glass transition temperature of PLA (grey bar), PLA/TT EO (light green bars) and $\mathrm{PLA} / \mathrm{M}$ EO (light pink bars) fibres as a function of the EO concentration. (For interpretation of the references to color in this figure legend, the reader is referred to the web version of this article.)

and PLA/M EO (light magenta bars) electrospun mats exhibited a lower $T_{g}$, which decreased with increasing EO concentration. Fibres produced from PLA solutions with 2.5 and $5.0 \% \mathrm{v} / \mathrm{v}$ of Tea Tree and Manuka EO were characterised by similar values of $T_{g}$ : around $35{ }^{\circ} \mathrm{C}$ and around $30{ }^{\circ} \mathrm{C}$ for 2.5 and $5.0 \% \mathrm{v} / \mathrm{v}$, respectively. For concentrations ranging from 7.5 to $15.0 \% \mathrm{v} / \mathrm{v}$, the difference in $T_{g}$ between PLA/TT EO and PLA/M EO fibres became statistically significant, with Tea Tree EO having a greater effect on $T_{g}$ than Manuka EO. When fibres were elecrospun from PLA solutions with 7.5, 10.0 and $15.0 \% \mathrm{v} / \mathrm{v}$ of Tea Tree EO, $T_{g}$ values of $(23 \pm 1)^{\circ} \mathrm{C},(15 \pm 1)^{\circ} \mathrm{C}$ and $(12 \pm 1)^{\circ} \mathrm{C}$ were recorded, respectively. On the other hand, for Manuka EO, $T_{g}$ dropped to $(19 \pm 1)^{\circ} \mathrm{C}$ when $15 \% \mathrm{v} / \mathrm{v}$ of oil was used. Values of $T_{g}$ in the range of $17-35{ }^{\circ} \mathrm{C}$ have been reported for PLA plasticised with low molecular weight molecules (less than $2000 \mathrm{~g} / \mathrm{mol}$ ), such as citrates or terpenes [34,35]. Tea Tree and Manuka oil are compounds with low molecular weight (lower than $500 \mathrm{~g} / \mathrm{mol}$ ) and rich in terpenes [2]. Like plasticisers, they are able to drastically lower the $T_{g}$ of PLA by enhancing polymer chain mobility and increasing polymer free volume [34,36,37]. The difference in thermal properties between PLA/TT EO and PLA/M EO fibres can be ascribed to the different chemical composition of the two essential oils, as previously demonstrated by Raman spectroscopy.

The thermal properties of the PLA/EOs mats had an impact on their morphology. As previously discussed (Fig. 1c, d and g), inter-fibre bonding was observed for high concentrations of Tea Tree and Manuka EO (10 and $15 \% \mathrm{v} / \mathrm{v}$ ). At those concentrations, the samples produced had $T_{g}$ in the range of $12-25^{\circ} \mathrm{C}$, which was comparable or slightly lower than room temperature (the electrospinning process was conducted at room temperature). As demonstrated in the literature, when electrospun fibres are annealed at a temperature close to their $T_{g}$, motions of the polymer chains at the fibre surface or in the bulk of the fibre, depending on the annealing temperature and polymer, give rise to fusion at the fibre-fibre junctions and eventually formation of collapsed structures [38-41]. Therefore, it can be assumed that PLA/TT EO mats exhibited more evident inter-fibre bonding than PLA/M EO fibres because their $T_{g}$ was much lower than the temperature at which the electrospinning process was carried out.

The plasticising effect of Tea Tree and Manuka oil on PLA also affected the mechanical properties of the composite fibres. PLA fibres exhibited tensile elongation at break $\left(\varepsilon_{b}\right)$ and strength at break $\left(\sigma_{b}\right)$ of $(0.3 \pm 0.1)$ and $(2.4 \pm 0.5) \mathrm{MPa}$, respectively, in agreement with previous studies (Fig. 4) [40,42]. The incorporation of Tea Tree and Manuka 
(a)

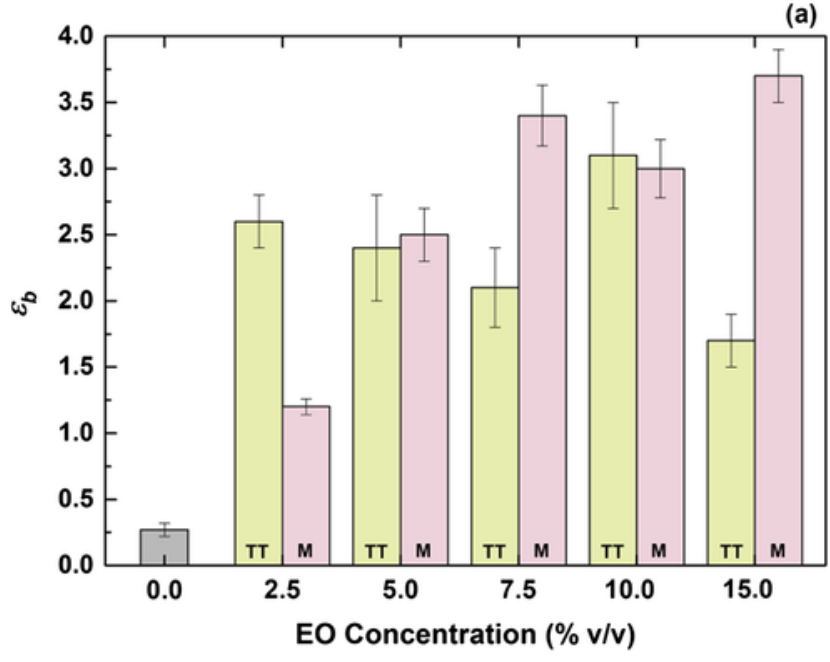

(b)

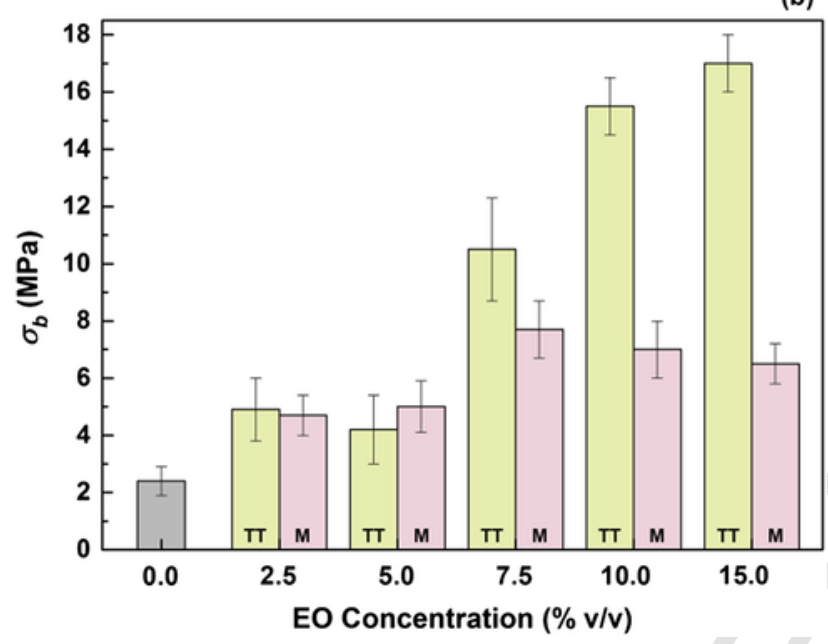

Fig. 4. (a) Elongation-at-break and (b) tensile strength of PLA (grey bar), PLA/TT EO (light green bars) and PLA/M EO (light pink bars) fibres as a function of the EO concentration. (For interpretation of the references to color in this figure legend, the reader is referred to the web version of this article.)

EOs resulted in a significant increase in both $\varepsilon_{b}$ and $\sigma_{b}$. For PLA/TT EO fibres (Fig. 4a), $\varepsilon_{b}$ reached values of $(2.6 \pm 0.2)$ when the lowest oil concentration was used $(2.5 \% \mathrm{v} / \mathrm{v})$, namely 8.7 times higher than the $\varepsilon_{b}$ of PLA fibres. Then, with increasing TT EO concentration (up to $10.0 \% \mathrm{v} / \mathrm{v}), \varepsilon_{b}$ remained almost constant with values between 2.1 and 3.1. However, a drop in $\varepsilon_{b}$ was recorded for fibrous mats produced from PLA solutions with $15.0 \% \mathrm{v} / \mathrm{v}$ of TT EO. While the high values of elongation at break (particularly for EO concentrations from 2.5 to $10.0 \% \mathrm{v} / \mathrm{v}$ ) are mainly due to the plasticising effect of TT EO, the decrease of $\varepsilon_{b}$ observed for $15.0 \% \mathrm{v} / \mathrm{v}$ concentration can be attributed to the morphology of the fibrous mat. Previous studies have found that incorporating EOs in PLA films leads to increase in elongation at break and the increase tends to be directly proportional to the amount of oil used, as expected for plasticising agents $[19,33,44]$. In the case of fibres electrospun from 15\% v/v PLA/TT EO solution, inter-fibre adhesion, shown in the morphological analysis in Fig. 1d, limits the sliding of fibres on top of each other during tensile deformation, resulting in a lower elongation. Similar behaviour has been observed, for example, for Polyethersulfone electrospun membranes after thermal treatment and for composite Polycaprolactone, collagen and elastin fibres $[45,46]$. Once tight cohesion between the fibres has been established, the elongation at break of the electrospun mat decreases, contrary to the tensile strength, which increases.

As shown in Fig. 4b, the addition of Tea Tree EO resulted in an increase of $\sigma_{b}$ : values of $(4.9 \pm 1.1) \mathrm{MPa}$ and $(4.2 \pm 1.2) \mathrm{MPa}$ were measured for fibres electrospun from PLA solutions with 2.5 and 5.0\% $\mathrm{v} / \mathrm{v}$ oil, respectively. The effect of Tea Tree EO on the $\sigma_{b}$ was much more evident for EO concentrations higher than $7.5 \% \mathrm{v} / \mathrm{v},(10.5 \pm 1.8)$ $\mathrm{MPa}$, with the highest value reported for concentration of $15.0 \% \mathrm{v} / \mathrm{v}$, $(17.0 \pm 1.0) \mathrm{MPa}$, as expected, due to the morphology of these mats. A previous study on thermally treated PLA electrospun membranes has demonstrated that, as a consequence of the temperature increase, the fibres undergo changes in morphology and fibre-fibre junctions become bonded [47]. Fibre coalescence increases as a function of the temperature together with the tensile strength of the membranes.

Tensile tests conducted on PLA/M EO electrospun samples also showed an increase of $\varepsilon_{b}$ and $\sigma_{b}$ with oil concentration (Fig. 4). Differently from PLA/TT EO fibres, $\varepsilon_{b}$ gradually increased from $(1.2 \pm 0.1)$ at $2.5 \% \mathrm{v} / \mathrm{v}$ EO concentration to $(3.4 \pm 0.2)$ at $5.0 \% \mathrm{v} / \mathrm{v}$. Then, $\varepsilon_{b}$ remained almost constant, with the highest value of $(3.7 \pm 0.2)$ at $15.0 \%$ $\mathrm{v} / \mathrm{v}$ EO concentration. The trend observed for $\sigma_{b}$ also differed from that of PLA/TT EO samples, with values in the range of 4.7-6.5 MPa, which were significantly lower than those of the PLA/TT EO samples, and without a large incremental change between the different EO concentrations. The lack of fibre coalescence in PLA/M EO mats can be the reason for the difference in mechanical properties observed for the two types of composite fibres, particularly at higher EO concentrations.

The antimicrobial properties of Tea Tree and Manuka EOs are attractive for preventing microorganism colonisation and biofilm formation $[8,48]$. As reported in literature, the mechanism of action of essential oils against bacteria is mainly due to the hydrocarbons partition into the bacteria membrane [49]. This induces damages to the cytoplasmic membranes, with disruption of its functions and eventually cell lysis. The effectiveness of the composite fibres produced was tested against S. epidermidis. This bacterium is abundant on human skin and often responsible for infections and formation of biofilms on indwelling medical devices [20]. As shown in Fig. 5, PLA mats without EO (Fig. 5a) and with Tea Tree oil (Fig. 5b) were not able to inhibit the proliferation of $S$. epidermidis, and the presence of the biofilm was visible in the agar medium. On the other hand, an inhibition zone of $2 \mathrm{~cm}$ in di- (a)

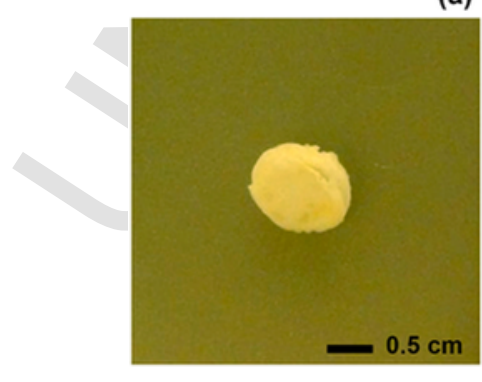

(b)

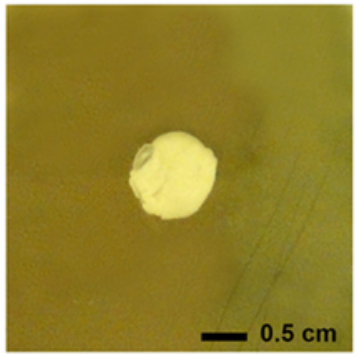

(c)

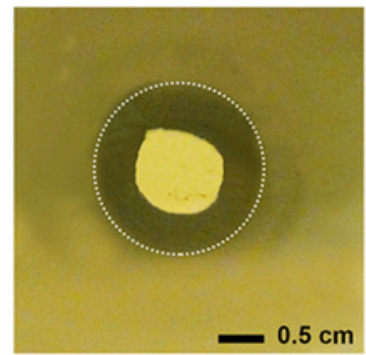

Fig. 5. Photographs of the in-vitro test for analysing the activity of (a) PLA, (b) PLA-TT EO (10\% v/v) and (c) PLA-M EO (10\% v/v) electrospun mats against S. epidermidis. 
ameter was detected for PLA fibres containing Manuka oil (Fig. 5c), indicating the antibacterial activity of those mats. Although Tea Tree oil is used for the treatment of skin infections induced by $S$. epidermidis [20], in this case its efficacy was limited. This was probably due to the low amount of terpinen-4-ol, and $\alpha$-terpinene available after the electrospinning process, as demonstrated by Raman spectroscopy. Conversely, Manuka oil was more stable during electrospinning, and the electrospun fibres still contained the compounds that confer antimicrobial properties on this essential oil (triketones) [31]. The glass transition temperature of the fibrous mats had also an effect on the release and diffusion of the active compounds inside the agar gel, leading to the inhibition zone observed. It is known that the use of plasticisers for polymer-based drug delivery systems promotes the release of bioactive agents [50].

\section{Conclusions}

In conclusion, it is demonstrated that essential oils of Tea Tree and Manuka can be used for enhancing the properties of PLA electrospun fibres, in terms of both mechanical and antibacterial characteristics. Chemical analysis of the fibres through Raman spectroscopy showed that the main components of Manuka oil were stable after the electrospinning process. This was different from Tea Tree oil in which a strong reduction in the intensity of the characteristic peaks was detected. Consequently the antibacterial activity of the composite PLA mats against $S$. epidermidis was affected. PLA/M EO fibres exhibited an inhibition effect against this pathogen, whereas PLA/TT EO mats were ineffective in preventing biofilm formation. Nevertheless, both EOs worked as plasticizers for PLA. In fact, the glass transition temperatures of PLA/TT EO and PLA/M EO fibres were reduced compared with the control PLA fibres. An increase in elongation-at-break as well as in tensile strength was observed for the composite fibres, and it was dependent on the EO concentration used. These results demonstrate that the PLA/EOs combinations studied have the potential to be exploited for the development of biomedical devices of which the mechanical behaviour and bioactivity can be controlled by using natural plant extracts.

\section{Uncited reference}

\section{Acknowledgements}

The authors acknowledge the use of the facilities and the assistance of K. Yendall in the Loughborough Materials Characterisation Centre, Department of Materials, for SEM analysis, and T. Coles from the Chemical Engineering Department of Loughborough University for the antibacterial tests.

\section{References}

[1] F. Reyes-Jurado, A. Franco-Vega, N. Ramirez-Corona, E. Palou, A. Lopez-Malo, Essential oils: antimicrobial activities, extraction methods, and their modelling, Food Eng. Rev. 7 (2008) 275-297.

[2] J.S. Raut, S.M. Karuppayil, A status review on the medicinal properties of essential oils, Ind. Crop. Prod. 62 (2014) 250-264.

[3] K.A. Rieger, J.D. Schiffman, Electrospinning an essential oil: cinnamaldehyde enhances the antimicrobial efficacy of chitosan/poly(ethylene oxide) nanofibers, Carbohydr. Polym. 113 (2014) 561-568.

[4] I. Liakos, L. Rizzello, H. Hajiali, V. Brunetti, R. Carzino, P.P. Pompa, A. Athanassiou, E. Mele, Fibrous wound dressings encapsulating essential oils as natural an timicrobial agents, J. Mater. Chem. B 3 (2015) 1583-1598.

[5] P. Wen, D.-H. Zhu, K. Feng, F.-J. Liu, W.-Y. Lou, N. Li, M.-H. Zong, H. Wu, Fabrication of electrospun polylactic acid nanofilm incorporating cinnamon essential oil/ $\beta$-cyclodextrin inclusion complex for antimicrobial packaging, Food Chem. 196 (2016) 996-1004.

[6] K. Balasubramanian, K.M. Kodam, Encapsulation of therapeutic lavender oil in an electrolyte assisted polyacrylonitrile nanofibres for antibacterial applications, RSC Adv. 4 (2014) 54892-54901.
[7] H. Hajiali, M. Summa, D. Russo, A. Armirotti, V. Brunetti, R. Bertorelli, A. Athanassiou, E. Mele, Alginate-lavender nanofibers with antibacterial and anti-inflammatory activity to effectively promote burn healing, J. Mater. Chem. B 4 (2016) 1686-1695.

[8] Z. Karami, I. Rezaeian, P. Zahedi, M. Abdollahi, Preparation and performance evaluations of electrospun poly( $\varepsilon$-caprolactone), poly(lactic acid), and their hybrid (50/ 50 ) nanofibrous mats containing thymol as an herbal drug for effective wound healing, J. Appl. Polym. Sci. 129 (2013) 756-766.

[9] T.V. Duncan, Applications of nanotechnology in food packaging and food safety: barrier materials, antimicrobials and sensors, J. Colloid Interface Sci. 363 (2011) $1-24$.

[10] D. Tartarini, E. Mele, Adult stem cell therapies for wound healing: biomaterials and computational models, Front. Bioeng. Biotechnol. 3 (2015) 206

[11] C.-H. Lee, S.-H. Chang, W.-J. Chen, K.-C. Hung, Y.-H. Lin, S.-J. Liu, M.-J. Hsieh, J.-H.S. Pang, J.-H. Juang, Augmentation of diabetic wound healing and enhancement of collagen content using nanofibrous glucophage-loaded collagen/PLGA scaffold membranes, J. Colloid Interface Sci. 439 (2015) 88-97.

[12] E. Mele, Electrospinning of natural polymers for advanced wound care: towards responsive and adaptive dressings, J. Mater. Chem. B 4 (2016) 4801-4812.

[13] W. Zhang, S. Ronca, E. Mele, Electrospun nanofibres containing antimicrobial plant extracts, Nano 7 (2017) 42.

[14] R. Morsy, M. Hosny, F. Reicha, T. Elnimr, Developing a potential antibacterial long-term degradable electrospun gelatin-based composites mats for wound dressing applications, React. Funct. Polym. 114 (2017) 8-12.

[15] J.-A. Park, S.-B. Kim, Preparation and characterization of antimicrobial electrospun poly(vinyl alcohol) nanofibers containing benzyl triethylammonium chloride, React. Funct. Polym. 93 (2015) 30-37.

[16] L. Yu, Y. Feng, Q. Li, X. Hao, W. Liu, W. Zhou, C. Shi, X. Ren, W. Zhang, PLGA/SF blend scaffolds modified with plasmid complexes for enhancing proliferation of endothelial cells, React. Funct. Polym. 91 (2015) 19-27.

[17] M.A. Souza, J.E. Oliveira, E.S. Medeiros, G.M. Glenn, L.H.C. Mattoso, Controlled release of linalool using nanofibrous membranes of poly(lactic acid) obtained by electrospinning and solution blow spinning: a comparative study, J. Nanosci. Nanotechnol. 15 (2015) 5628-5636.

[18] C.L.S. de Oliveira Mori, N.A. dos Passos, J.E. Oliveira, T.F. Altoé, F.A. Mori, L.H. Capparelli Mattoso, J.R. Scolforo, G.H. Denzin Tonoli, Nanostructured polylactic acid/Candeia essential oil mats obtained by electrospinning, J. Nanomater. 2015 (2015) 1-9.

[19] Y. Qin, W. Li, D. Liu, M. Yuan, L. Li, Development of active packaging film made from poly (lactic acid) incorporated essential oil, Prog. Org. Coat. 103 (2017) $76-82$.

[20] M. Otto, Staphylococcus epidermidis-the 'accidental' pathogen, Nat. Rev. Microbiol. 7 (2009) 555-567.

[21] R. Casasola, N.L. Thomas, A. Trybala, S. Georgiadou, Electrospun poly lactic acid (PLA) fibres: effect of different solvent systems on fibre morphology and diameter, Polymer 55 (2014) 4728-4737.

[22] R. Casasola, N.L. Thomas, S. Georgiadou, Electrospinning of poly(lactic acid): theoretical approach for the solvent selection to produce defect-free nanofibers, J. Polym. Sci. B Polym. Phys. 54 (2016) 1483-1498.

[23] D.-W. Sun, Modern Techniques for Food Authentication, 1st Ed., Elsevier, New York, 2008161-163.

[24] F. Christoph, K.-H. Kubeczka, E. Stahl-Biskup, The composition of commercial manuka oils from New Zealand, J. Essent. Oil Res. 11 (1999) 705-710.

[25] N.G. Porter, A.L. Wilkins, Chemical, physical and antimicrobial properties of essential oils of Leptospermum scoparium and Kunzea ericoides, Phytochemistry 50 (1999) 407-415.

[26] N. Wu, Y. Wang, Y. Lei, B. Wang, C. Han, Y. Gou, Q. Shi, D. Fang, Electrospun interconnected $\mathrm{Fe}-\mathrm{N} / \mathrm{C}$ nanofiber networks as efficient electrocatalysts for oxygen reduction reaction in acidic media, Sci Rep 5 (2015) 17396.

[27] T.E. Kerr-Phillips, V. Woehling, R. Agniel, G.T.M. Nguyen, F. Vidal, P. Kilmartin, C. Dric Plesse, J. Travas-Sejdic, Electrospun rubber fibre mats with electrochemically controllable pore sizes, J. Mater. Chem. B 3 (2015) 4249-4258.

[28] G. Kister, G. Cassanas, M. Vert, Effects of morphology, conformation and configuration on the IR and Raman spectra of various poly(lactic acid)s, Polymer 39 (1998) 267-273.

[29] K. Bruckmoser, K. Resch, Effect of processing conditions on crystallization behavior and mechanical properties of poly(lactic acid) staple fibers, J. Appl. Polym. Sci. 132 (2015) 42432. 
[30] D.P. Killeen, J.W. van Klink, B.M. Smallfield, K.C. Gordon, N.B. Perry, Herbicidal $\beta$-triketones are compartmentalized in leaves of Leptospermum species: localization by Raman microscopy and rapid screening, New Phytol. 205 (2015) 339-349.

[31] D.P. Killeen, L. Larsen, F.E. Dayan, K.C. Gordon, N.B. Perry, J.W. van Klink, Nortriketones: antimicrobial trimethylated acylphloroglucinols from manuka (Leptospermum scoparium), J. Nat. Prod. 79 (2016) 564-569.

[32] P.V. Jentzsch, L.A. Ramos, V. Ciobota, Handheld Raman spectroscopy for the distinction of essential oils used in the cosmetics industry, Cosmetics 2 (2015) $162-176$.

[33] S. Pirani, H.M.N. Abushammala, R. Hashaikeh, Preparation and characterization of electrospun PLA/nanocrystalline cellulose-based composites, J. Appl. Polym. Sci. 130 (2013) 3345-3354.

[34] T. Mekonnen, P. Mussone, H. Khalilb, D. Bressler, Progress in bio-based plastics and plasticizing modifications, J. Mater. Chem. A 1 (2013) 13379-13398.

[35] M.P. Arrieta, J. López, S. Ferrándiz, M.A. Peltzer, Characterization of PLA limonene blends for food packaging applications, Polym. Test. 32 (2013) 760-768.

[36] V. Nagarajan, A.K. Mohanty, M. Misra, Perspective on polylactic acid (PLA) based sustainable materials for durable applications: focus on toughness and heat resistance, ACS Sustain. Chem. Eng. 4 (2016) 2899-2916.

[37] M. Gurgel Adeodato Vieira, M. Altenhofen da Silva, L. Oliveira dos Santos, M. Masumi Beppu, Natural-based plasticizers and biopolymer films: a review, Eur. Polym. J. 47 (2011) 254-263.

[38] S.J. Lee, S.H. Oh, J. Liu, S. Soker, A. Atala, J.J. Yoo, The use of thermal treatments to enhance the mechanical properties of electrospun poly( $\varepsilon$-caprolactone) scaffolds, Biomaterials 29 (2008) 1422-1430.

[39] S.-S. Choi, S.G. Lee, C.W. Joo, S.S. Im, S.H. Kim, Formation of interfiber bonding in electrospun poly(etherimide) nanofiber web, J. Mater. Sci. 39 (2004) 1511-1513.

[40] Y. You, S.W. Lee, S.J. Lee, W.H. Park, Thermal interfiber bonding of electrospun poly(L-lactic acid) nanofibers, Mater. Lett. 60 (2006) 1331-1333.

[41] L. Mascia, R. Su, J. Clarke, Y. Lou, E. Mele, Fibres from blends of epoxidized nat- ural rubber and polylactic acid by the electrospinning process: compatibilization and surface texture, Eur. Polym. J. 87 (2017) 241-254.

[42] Q. Shi, C. Zhou, Y. Yue, W. Guo, Y. Wu, Q. Wu, Mechanical properties and in vitro degradation of electrospun bio-nanocomposite mats from PLA and cellulose nanocrystals, Carbohydr. Polym. 90 (2012) 301-308.

[43] M. Llana-Ruiz-Cabello, S. Pichardo, J.M. Bermúdez, A. Baños, C. Núñez, E. Guillamón, S. Aucejo, A.M. Cameán, Development of PLA films containing oregano essential oil (Origanum vulgare L. virens) intended for use in food packaging, Food Addit. Contam., Part A 33 (2016) 1-13.

[44] J. Ahmed, M.Z. Mulla, Y.A. Arfat, Thermo-mechanical, structural characterization and antibacterial performance of solvent casted polylactide/cinnamon oil composite films, Food Control 69 (2016) 196-204.

[45] S. Homaeigohar, J. Koll, E.T. Lilleodden, M. Elbahri, The solvent induced interfiber adhesion and its influence on the mechanical and filtration properties of polyethersulfone electrospun nanofibrous microfiltration membranes, Sep. Purif. Technol. 98 (2012) 456-463.

[46] R. Erdem, M. Yüksek, E. Sancak, O. Atak, M. Erginer, L. Kabasakal, A. Beyit, Electrospinning of single and multilayered scaffolds for tissue engineering applications, J. Text. Inst. 108 (2017) 935-946.

[47] S. Ramaswamy, L.I. Clarke, R.E. Gorga, Morphological, mechanical, and electrical properties as a function of thermal bonding in electrospun nanocomposites, Polymer 52 (2011) 3183-3189.

[48] F. Kayaci, O.C.O. Umu, T. Tekinay, T. Uyar, Antibacterial electrospun poly (lactic acid) (PLA) nanofibrous webs incorporating triclosan/cyclodextrin inclusion complexes, J. Agric. Food Chem. 61 (2013) 3901-3908.

[49] C.F. Carson, K.A. Hammer, T.V. Riley, Melaleuca alternifolia (tea tree) oil: a review of antimicrobial and other medicinal properties, Clin. Microbiol. Rev. 19 (2006) $50-62$.

[50] F. Alexis, Factors affecting the degradationand drug-release mechanism of poly(lacticacid) and poly[(lactic acid)-co-(glycolic acid)], Polym. Int. 54 (2005) 36-46. 